\title{
The Relationship between Achievement Motivation and Entrepreneurial Talent in Nursing Students of Gonabad University of Medical Sciences
}

\author{
Habib Shareinia ${ }^{1}$, Afsaneh Noorayi ${ }^{2}$, Mahdi Esmaeilnia ${ }^{2}$, Batool \\ Mohamadian ${ }^{3}$, Reza Esmaeili ${ }^{4}$, Saeed Erfanpoor ${ }^{\text {** }}$
}

\author{
${ }^{1}$ Faculty Member, Department of Medical-Surgical Nursing, Faculty of Nursing and Midwifery, \\ Social Development and Health Promotion Research Centre, Gonabad University of Medical \\ Sciences, Gonabad, Iran \\ ${ }^{2}$ BSc Student Nursing, Student Research Committee, Gonabad University of Medical Sciences, \\ Gonabad, Iran \\ ${ }^{3}$ Faculty Member, Department of Operating Room, Faculty of Paramedical, Gonabad University of \\ Medical Sciences, Gonabad, Iran \\ ${ }^{4}$ Assistant Professor, Department of Public Health, Faculty of Health, Gonabad University of Medical \\ Sciences, Gonabad, Iran \\ ${ }^{5}$ Faculty Member, Department of Social Medicine, Faculty of Health, Gonabad University of Medical \\ Sciences, Gonabad, Iran \\ * Corresponding author: Saeed Erfanpoor, Faculty Member, Department of Social Medicine, Faculty \\ of Health, Gonabad University of Medical Sciences, Gonabad, Iran. E-mail: \\ saeed.erfanpoor@yahoo.com
}

Received: 14 Jun 2017

Accepted: 17 Oct 2017

\begin{abstract}
Introduction: Skilled manpower education for the society is one of the main objectives of higher education in Iran. Nowadays, since unemployment is one of the biggest economic problems in Iran, higher education has a dual mission of employment and development of entrepreneurial talent. Furthermore, achievement motivation is significantly important as a success factor, especially in nursing students. Therefore, this study was conducted with the aim of determining the relationship between achievement motivation and entrepreneurial talent in nursing students of Gonabad University of Medical Sciences.

Methods: This study was conducted by cross-sectional descriptive-analytical method. The statistical population included all 160 second to eighth semester nursing students, who were selected by the census method from Gonabad University of Medical Sciences. Inclusion criteria included studying at the time of the research and the students' willingness to participate in the study. The exclusion criterion was failure to complete the questionnaire. Data collection tools of this study included the demographic information questionnaire, entrepreneurial talent measurement questionnaire by Kordnaeij et al., and Hermans' achievement motivation questionnaire. The collected data were analyzed using descriptive statistic and Pearson's test. Data analysis was done using the SPSS 20 software.
\end{abstract}

Results: The status of entrepreneurial talent was weak in the majority of students (70\%), and $93.1 \%$ of research units had high achievement motivation. According to the results of the Pearson test, there was a significant positive relationship between achievement motivation and entrepreneurial talent $(\mathrm{P}<0.001$ and $\mathrm{r}=0.332)$.

Conclusions: Students' weak entrepreneurial capacity indicates the necessity of planning and concentration on reinforcement of factors, which affect entrepreneurial capacity in curricula and educational assistance curricula.

Keywords: Entrepreneurial Talent, Achievement Motivation, Nursing Students 


\title{
بررسى ارتباط انكيزه يشرفت و استعداد كار آفرينى در دانشجويان يرستارى دانشكاه علوم يزشكى كناباد
}

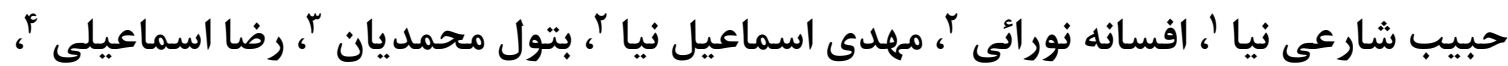 \\ سعيد عرفان يور هـ
}

عضو هيات علمى، كروه يرستارى داخلى جراحى، دانشكده يرستارى و مامايى، مركز تحقيقات توسعه اجتماعى و ارتقاى سلامت، دانشكاه علوم يزشكى كناباد، كناباد، ايران

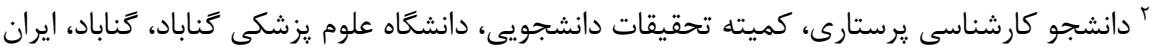

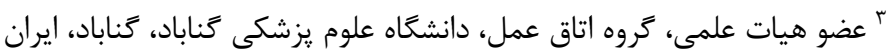

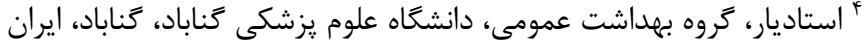

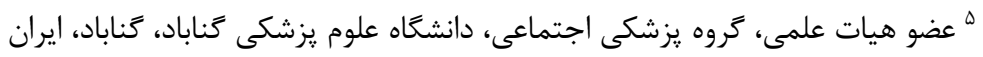

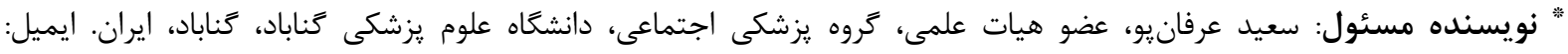
saeed.erfanpoor@yahoo.com

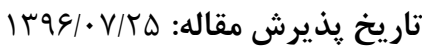

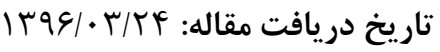

مقدمه: يكى از اهداف مهم نظام آموزش عالى كشور، يرورش نيروى انسانى متخصص و مورد نياز جامعه است. در حال حاض حاضر با توجه

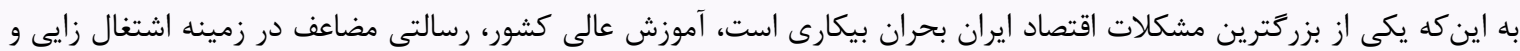

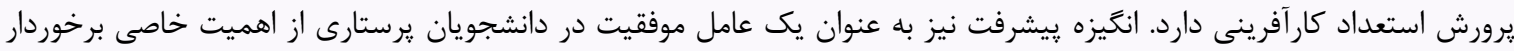

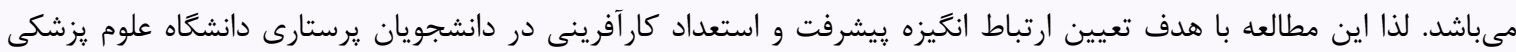
كناباد انجام يذيرفت.

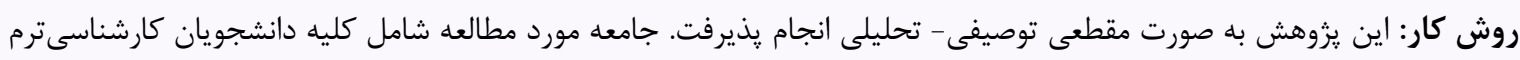

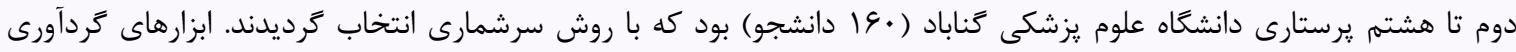

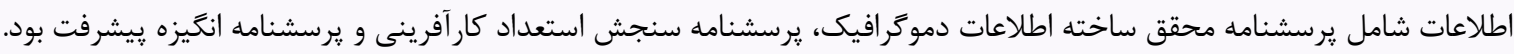

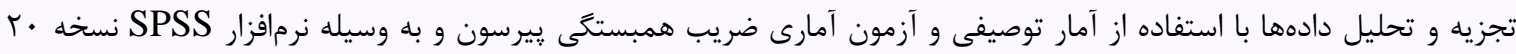
انجام يذيرفت.

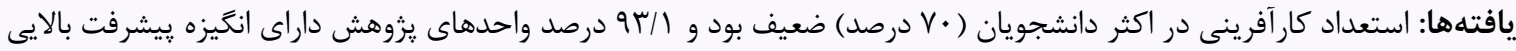

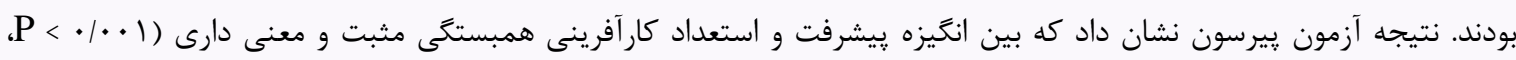
332/0 = r

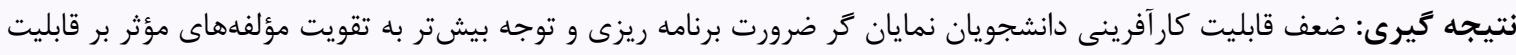

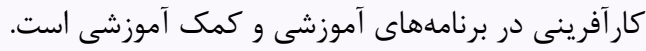

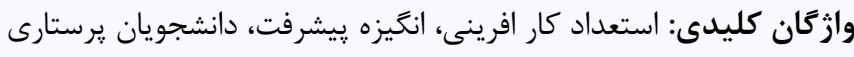
تمامى حقوق نشر براى انجمن علمى يرستارى ايران محفوظ است.

آميز همجون خوداشتغالى، ايجاد يك سازمان جديد و يا توسعه

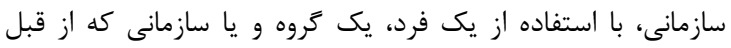

كارآفرينى در عمل توانايى خلق يك جيز از هيج است. به عبارتى هر

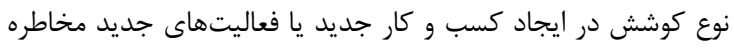


استعداد كارآفرينى، انَّيزه بِيشرفت و ارتباط اين دو با يكديخر در

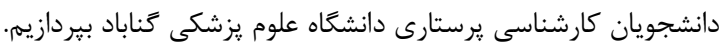

\section{روش كار}

اين يزوهش به صورت مقطعى توصيفى - تحليلى انجام يذيرفت. جامعه

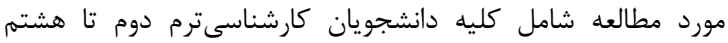

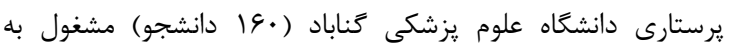

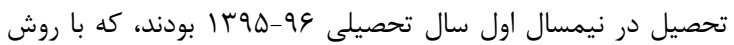

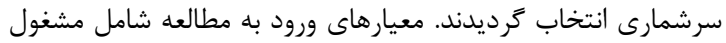

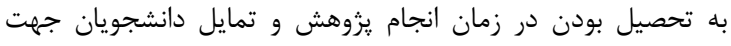

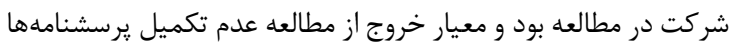

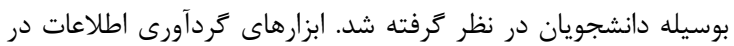

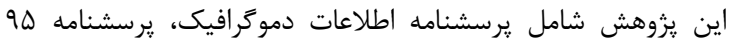

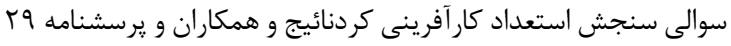

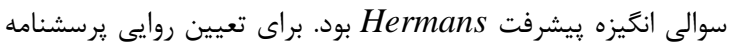

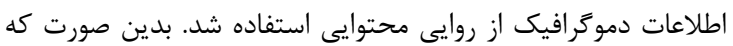

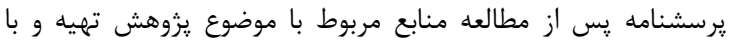

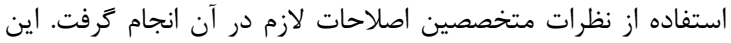

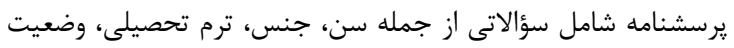

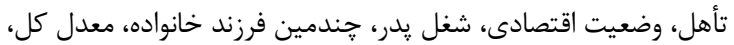

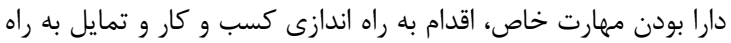

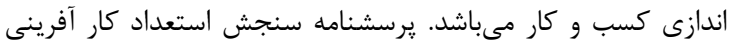

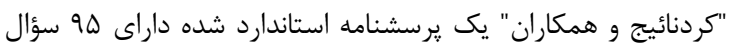

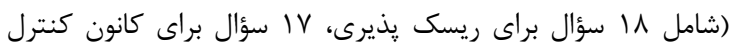

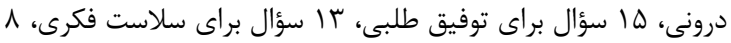

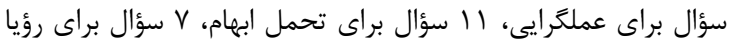

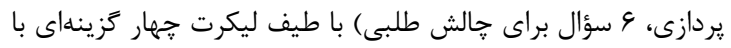

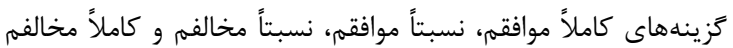

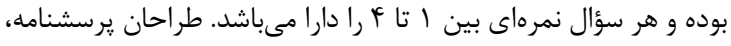

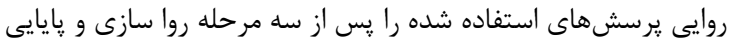

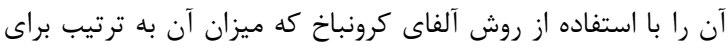

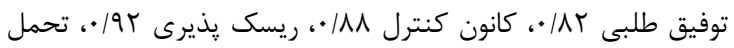

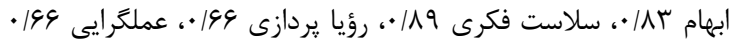

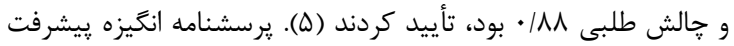
Hermans

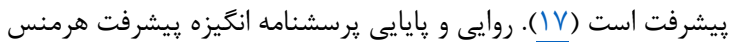

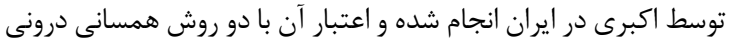

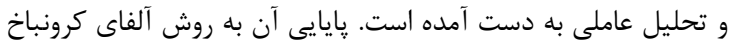

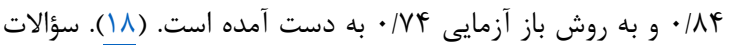

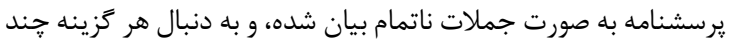
جمله آورده شده است. جهت يكسان سازى ارزش سؤالات براى هر هو

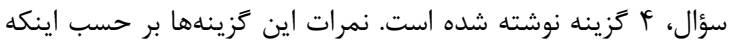

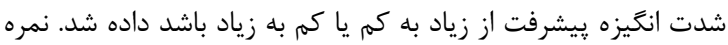

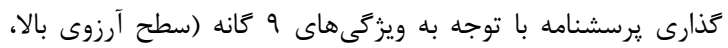

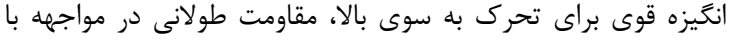

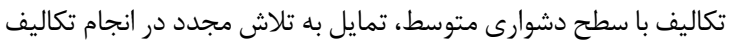

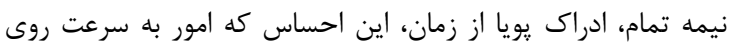

تأسيس شده، كار آفرينى ناميده مىشود ( (). كار آفرينى موضوعى است

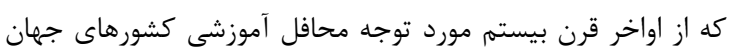

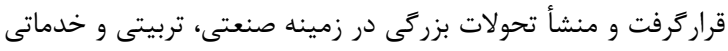

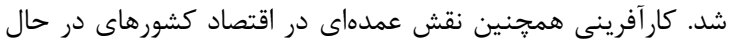

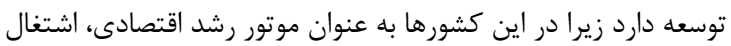

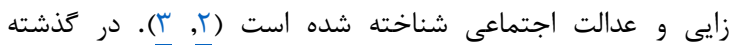

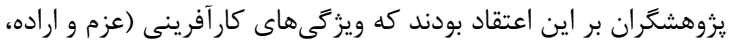

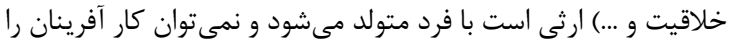

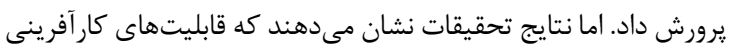

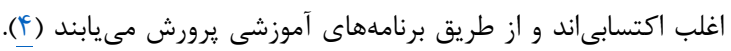

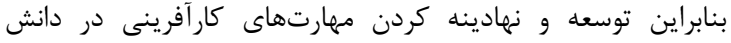

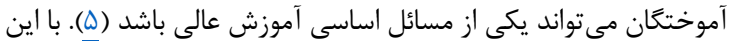

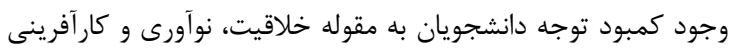

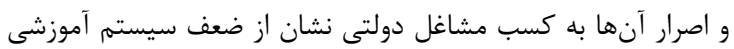

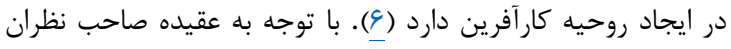

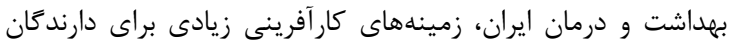

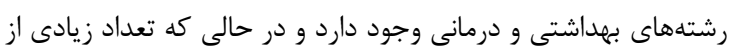

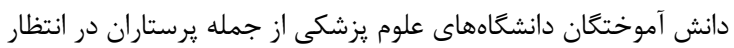

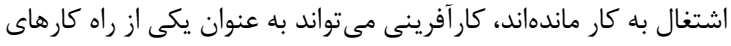

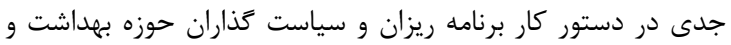

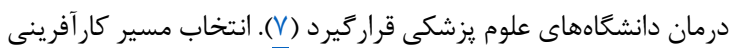

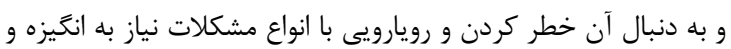

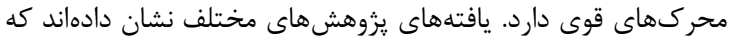

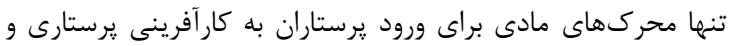

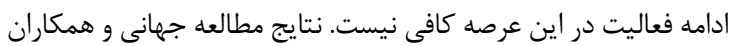

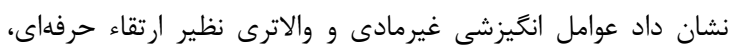

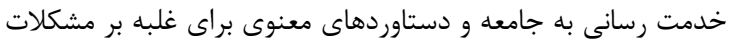

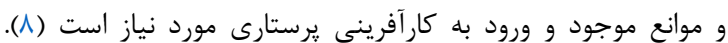

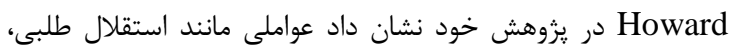

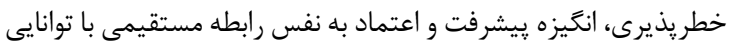

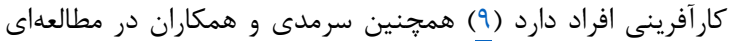

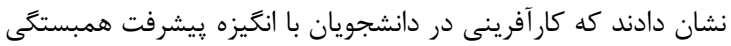

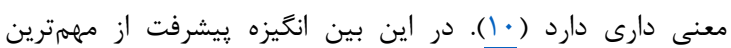

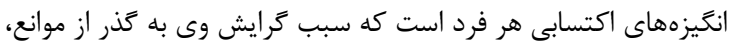

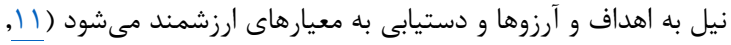

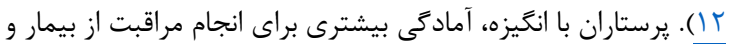

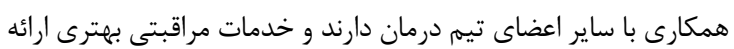

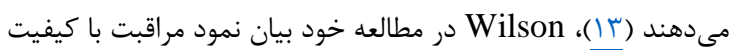

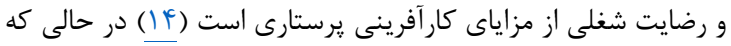

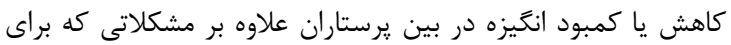

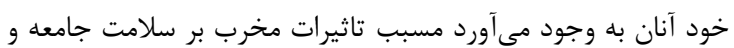

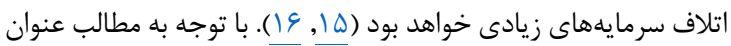

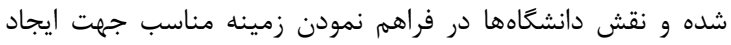

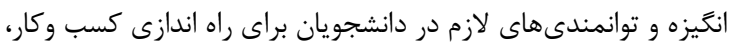

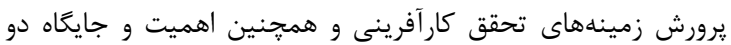

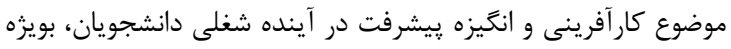

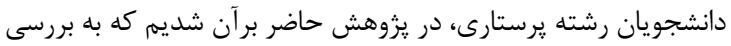


درصد) آن ها مرد بودند كه ميانخين و انحراف معيار سنى آنها به ترتيب

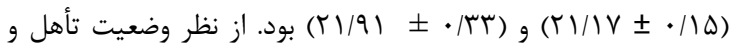

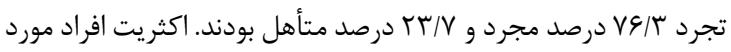

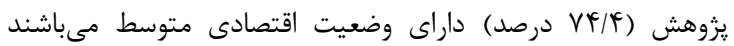

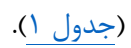

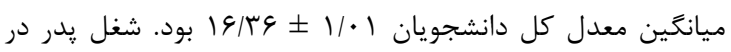

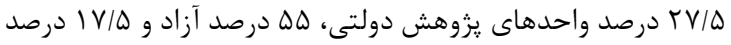

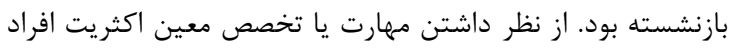

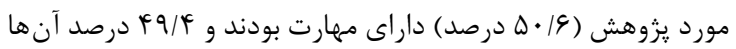

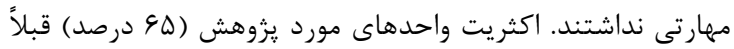

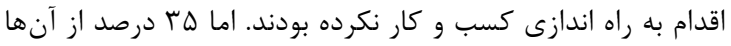

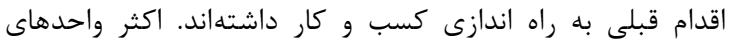

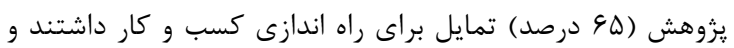

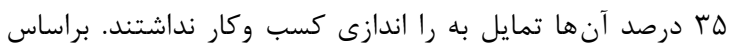

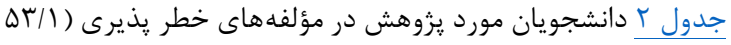

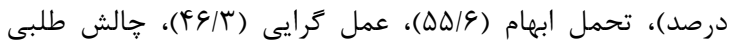

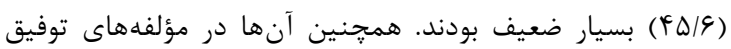

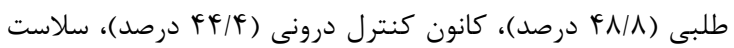

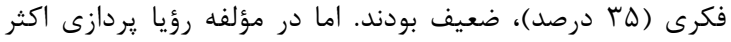

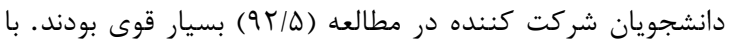

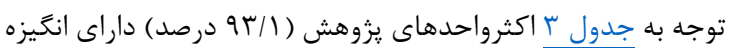

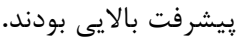

مىدهند، آينده نتَرى، توجه به ملاك شايستخى در انتخاب دوست،

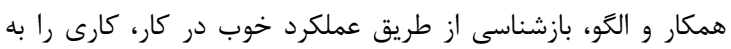

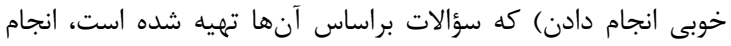

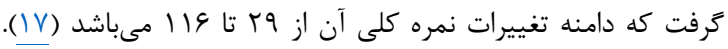

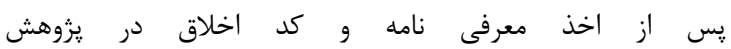
از معاونت يزوهشى دانشخاه علوم

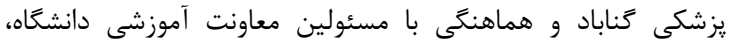

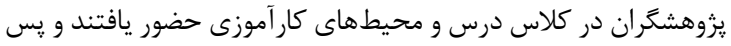

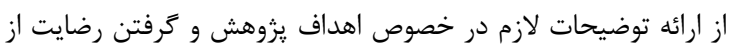

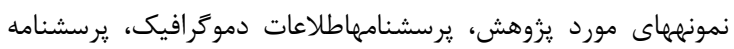

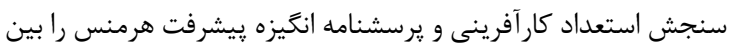

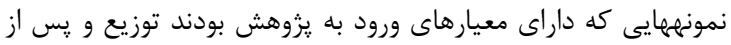

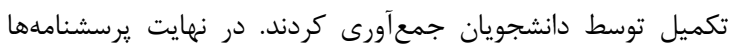

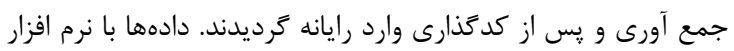
spss توصيفى دادهاى كمى بصورت ميانخين \pm انحراف معيار و دادههاى تصديل

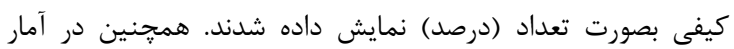

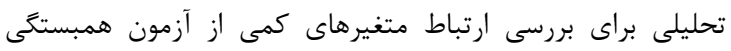
ييرسون (Pearson Correlation) استفاده كرديد.

يافته ها

يافتهاى اين يزوهش نشان داد از مجموع • •1 نفر دانشجوى برستارى

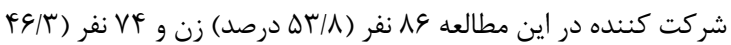

جدول ا: وضعيت اقتصادى دانشجويان شركت كننده در يزوهش

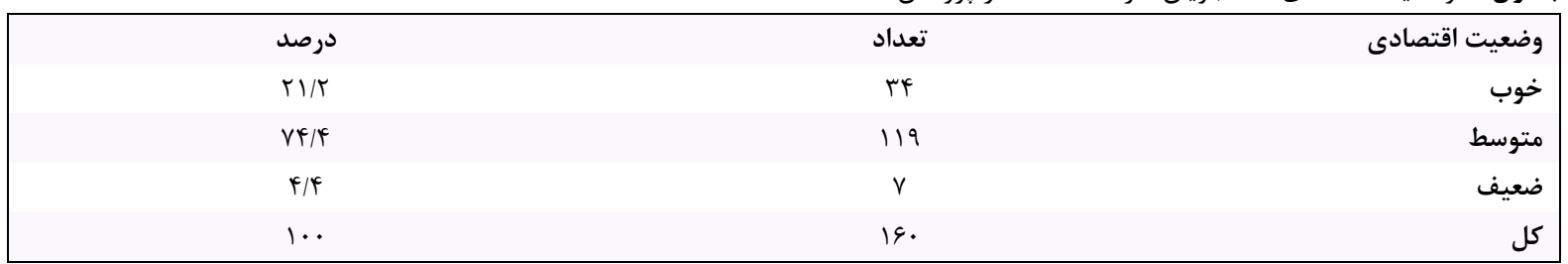

جدول r: توزيع فراوانى مطلق و نسبى قابليت كار آفرينى و مؤلفههاى آن

\begin{tabular}{|c|c|c|c|c|}
\hline بسيارقوى & قوى & ضعيف & بسيارضعيف & ويزَى \\
\hline $9(\Delta / \&)$ & $r \Delta(\mid \Delta / \varepsilon)$ & $V \wedge(F \wedge / \Lambda)$ & $\boldsymbol{F} \wedge(\Gamma \cdot)^{*}$ & توفيق طلبى \\
\hline$r \mid(I r / I)$ & $r \Delta(r \backslash / q)$ & $V I(F F / F)$ & $M r(T \cdot 19)$ & كانون كنترل درونى \\
\hline$q(\Psi / \Lambda)$ & $r \cdot(\mid N / \Lambda)$ & $r q(Y F / F)$ & $\Lambda \Delta(\Delta r / I)$ & خطر يذيرى \\
\hline$\wedge(\Delta)$ & $r F(\mid Q)$ & $r q(Y K / \mathcal{F})$ & $\Lambda 9(\Delta \Delta / \varphi)$ & تحمل ابهام \\
\hline $\operatorname{rF}(\mid \Delta)$ & $\operatorname{Fr}(T \& / T)$ & $\Delta \mathcal{G}(\Gamma \Delta)$ & $\Gamma \wedge(T r / \Lambda)$ & سلاست فكرى \\
\hline $\operatorname{lF} \wedge(9 Y / \Delta)$ & $1 \cdot(g / \pi)$ & $1(\cdot / 9)$ & $1(\cdot / 9)$ & رؤيايردازى \\
\hline $19(1 \cdot)$ & $r q(\mid N / 1)$ & $f \mid(T \Delta / \varphi)$ & $V F(\uparrow \& / \pi)$ & عملكَرايى \\
\hline$\varphi(\Gamma / \Lambda)$ & $11(8 / 9)$ & $V \cdot(\mathcal{K} / \Lambda)$ & $V \Psi(F \Delta / \varphi)$ & جالش طلبى \\
\hline
\end{tabular}

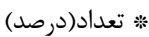

جدول با: توزيع فراوانى مطلق و نسبى انگيزه بِيشرفت

\begin{tabular}{|c|c|c|}
\hline درصد & تعداد & انغيزه بِيشرفت \\
\hline $9 / 9$ & 11 & انگيزه كم \\
\hline $9 \% / 1$ & 149 & انگيزه بالا \\
\hline $1 \ldots$ & 19. & كل \\
\hline
\end{tabular}


جدول f: همبستگى بين نمره كلى يرسشنامه انكَيزه پِيشرفت هرمنس با نمره كلى يرسشنامه سنجش استعداد كار آفرينى

\begin{tabular}{|c|c|c|c|}
\hline P-value & $\mathbf{R}$ & نمره كل "برسشنامه" & متغير \\
\hline$<\cdot / \cdot \cdot 1$ & ( & $\wedge \Gamma / \wedge \wedge \pm 9 / \wedge q$ & انغيزه ييشرفت \\
\hline$<\cdot|\cdot \cdot|$ & צזא/. & $1 / M 1 \pm 1 / \cdot r$ & استعداد كار آفرينى \\
\hline
\end{tabular}

ميانخين土 انحراف معيار

ويزگگىهاى كارآفرينى دانشجويان در سطح بسيار قوى بود و مطالعه

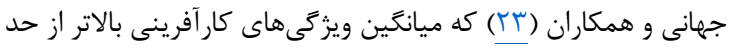

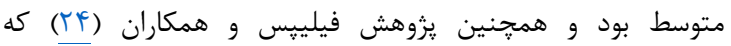

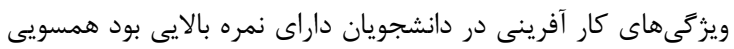

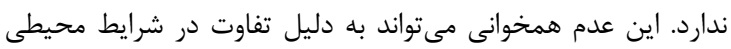

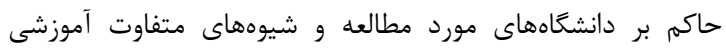
دانشجويان باشد.

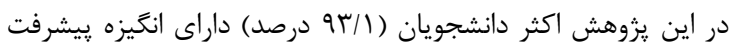

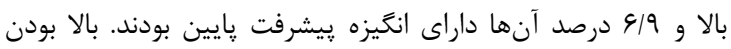

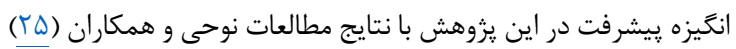

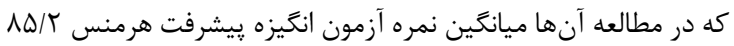

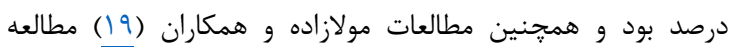

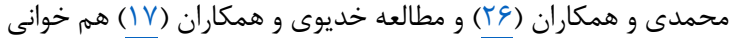

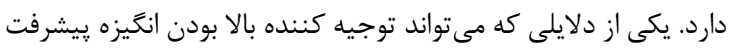

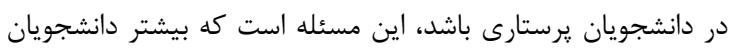

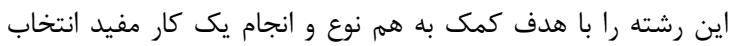

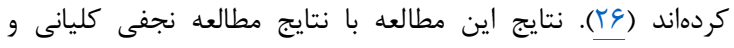

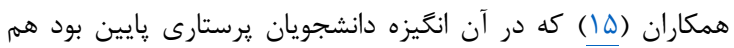

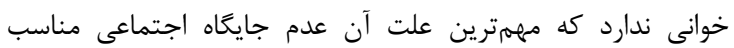

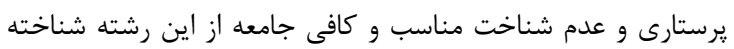

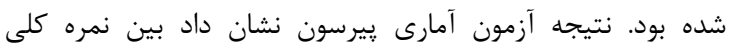

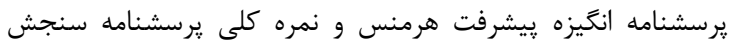

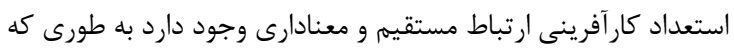

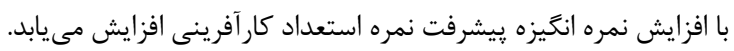

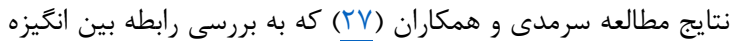

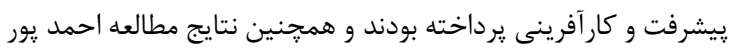

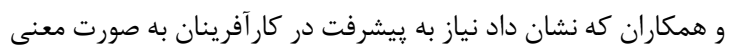

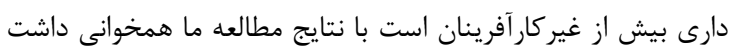

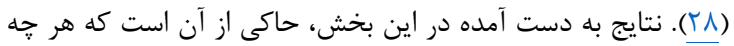

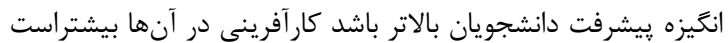

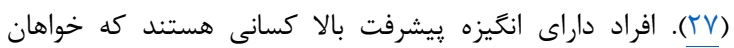

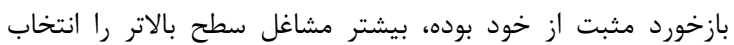

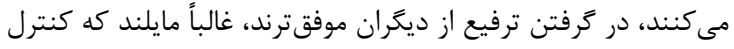

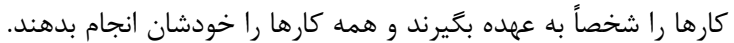
همجنين غالباً در انجام وظايف انفرادى و مستقل و و راه انداختن كارن كارها به صورت فردى موفقتر هستند تا كار كروهى كه با توجه به اين شرايط

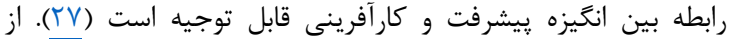

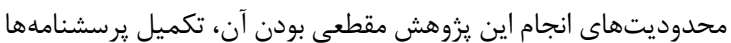

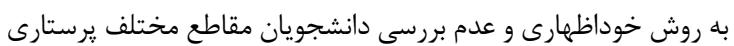

همجنين نتيجه آزمون همبستخى يِيرسون نشان داد بين نمره كلى

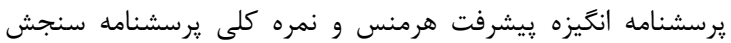

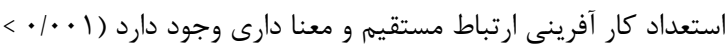
P

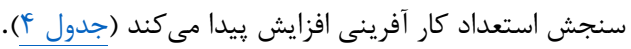

\section{بحث}

يروهش حاضر با هدف تعيين ميزان استعداد كار آفرينى، انخيزه پِيشرفت

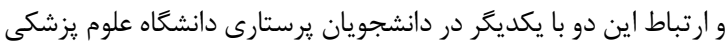

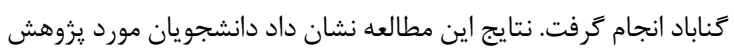

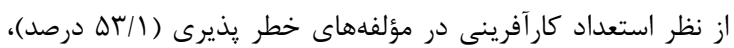

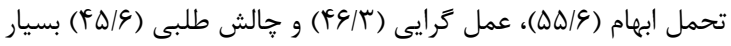

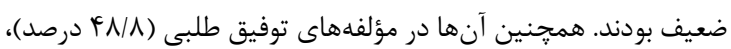

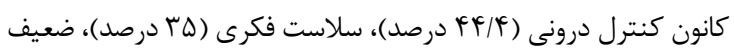

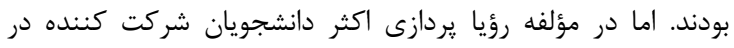

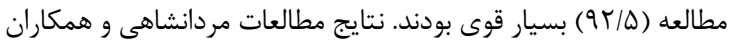

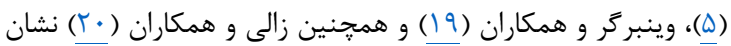

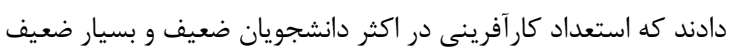

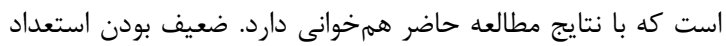

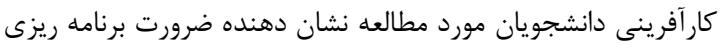

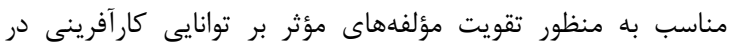

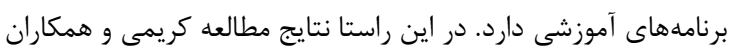

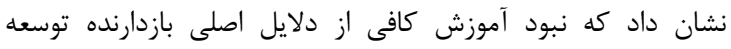

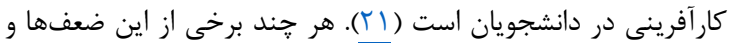

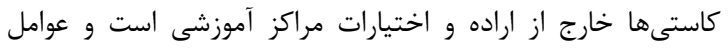

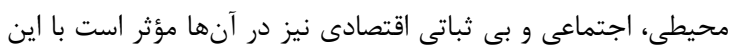

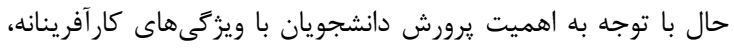

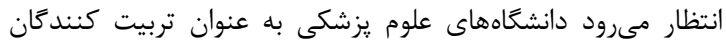

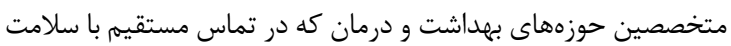

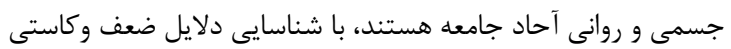

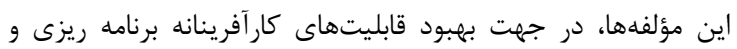

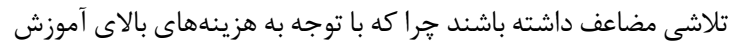

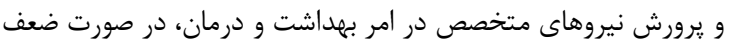

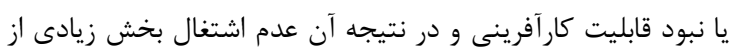

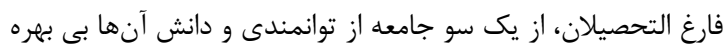

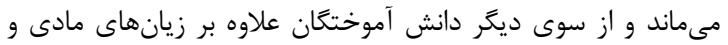

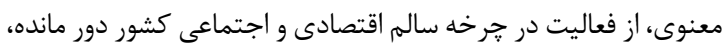

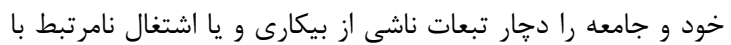

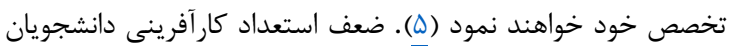

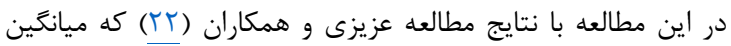




$$
\begin{aligned}
& \text { يزوهش مسئولان آموزش ثرستارى مىبايست راهبردهاى ايجاد و ارتقاء }
\end{aligned}
$$

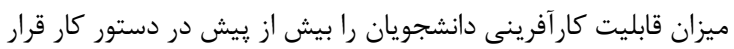

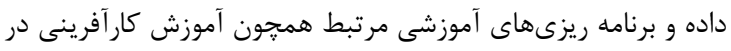

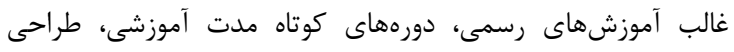

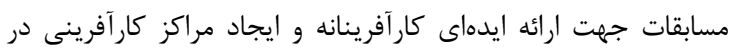

$$
\begin{aligned}
& \text { دانشكدهاى يرستارى را به انجام رساند. } \\
& \text { سباسگزןارى }
\end{aligned}
$$

از تمامى دانشجويانى كه ما را در تكميل يرسشنامهایى اين يزوهش

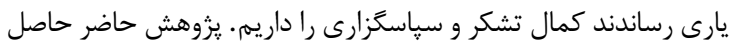

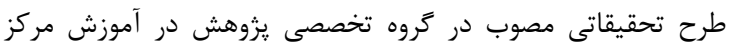

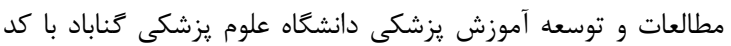
طرح EDC/2/1395 است كه بدينوسيله از مسئولين و كارشناسان آن مركز تشكر و قدردانى به عمل مى آوريم.

\section{References}

1. Kamaliyan A, Fazel A. The relationship between emotional intelligence and entrepreneurship students. Entrepreneurship Dev. 2012;3(11):127-46.

2. Shoja-Ghale-Dokhtar L, Rahimean H, Jarahi L. Evaluation of entrepreneurship capabilities in students of Mashhad University of Medical Sciences. J Med Educ Dev. 2014;9(3):2-9.

3. Kiyanymavi N, Kiyanymavi R. Check and compare the entrepreneurs' willingness male and female students of physical education And provide a model for measuring interest in entrepreneurship. J Manage Dev Transform. 2012;9:19-26.

4. Jahani N, Alaghmand A, Mirjood H. Evaluation of entrepreneurship attributes in dental students at babol medical university. Iranian $\mathrm{J}$ Med Educ. 2013;5(13):413-20.

5. Mardanshahy M, Tajik Esmaili A, Mobaraky M. Entrepreneurial talent search outgoing and incoming students at public universities. J Entrepreneursh Dev. 2014;7(14):755-71.

6. Anderson M. The complex relations between the academy and industry: views form the literature. $\mathrm{J}$ Higher Educ. 2001;72(2):244-6.

7. Mardanshahi M, Dolati A. Compare of entreprenrurial abilities in students of Mazandaran universityof Medical sciences. Iranian $\mathrm{J}$ Med Educ. 2015;15(48):381-90.

8. Jahani S, Abedi H, Fallahi Khoshknab M, Elahi N. Perceived entrepreneurial motivators by Iranian nurse entrepreneurs: a qualitative study. Q J Nurs Manage. 2014;3(3):68-77.

9. Howard S. Developing entrepreneurial potential in youth: The effects of entrepreneurial education and venture creation. 4th ed. Florida University of South Florida publication; 2014.

10. Sarmadi M, Mahboobi T, Abdollahzade $H$. Investigating the Relationship between of Intelligence Beliefs, Self-Esteem, Achievement Motive and Entrepreneurship among the Students of Payame Noor University in Western and Eastern Azerbaijan. J Mod Psychol Res. 2009;4(14):63-88.

$$
\begin{aligned}
& \text { بود. بِيشنهاد مى گردد يزوهش هايى مشابه به صورت طولى و در مقاطع }
\end{aligned}
$$

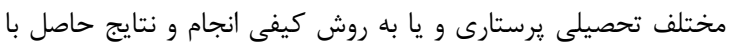

$$
\begin{aligned}
& \text { يكديگر مقايسه تردند. } \\
& \text { نتيجه تيرى }
\end{aligned}
$$

نتايج اين يزوهش نشان داد كه دانشجويان مورد مطالعه از نظر قابليت

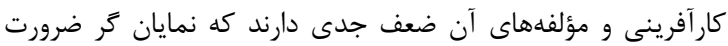

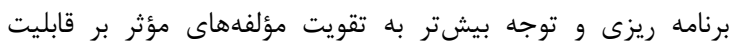

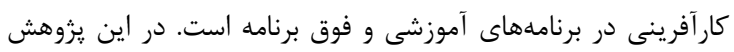

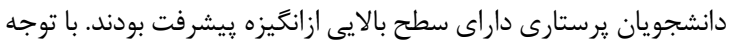

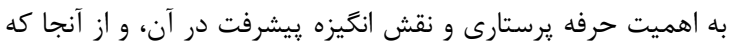

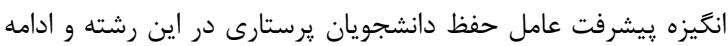

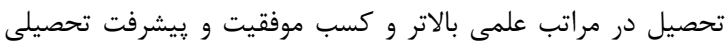

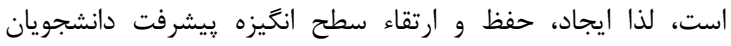

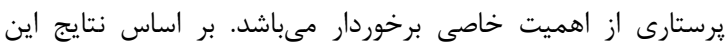

11. Molazade A, Hamayeli Mehrabani H, Gholami M, Mortazavi A, Dowlatkhah H, GHodsi R, et al. Relationship between the spiritual intelligence ,academic achievement, and achievement motivation among the students of fasa university of medical sciences in 2013. Commun Health Res. 2013;7(23):59-64.

12. Molazadeh A, Hamayelimehrabani H, Gholami M, Mortazavi A, Dolatkhah H, Ghodsi R. Relationship of general health and improvement motivation with educational success among the students of Fasa University of Medical Sciences in 2013. Fac Med Sci Nishapur. 2014;2(4):54-61.

13. Toode K, Routasalo P, Suominen T. Work motivation of nurses: a literature review. Int $\mathrm{J}$ Nurs Stud. 2011;48(2):246-57.

DOI: 10.1016/j.ijnurstu.2010.09.013 PMID: 20947085

14. Wilson A, Averis A, Walsh K. The influences on and experiences of becoming nurse entrepreneurs: a Delphi study. Int J Nurs Pract. 2003;9(4):236-45. PMID: 12887375

15. Najafi Kalyani M, Sharif F, Moattari M, Gamshidi N, Karimi S. Decrease in motivation of nursing students: A qualitative study. Nurs Educ. 2011;6(21):39-47.

16. Valizadeh S, Hririyan H. Nurses Work Motivation: A Big Challenge for Health System; a Review article. J Prev Care Nurs Midwifery. 2015;5(2):56-64.

17. Khadivi A, Vakilh Mafakheri A. The relationship between achievement motivation, locus of control, self-concept and academic achievement the first year of high school five districts of tabriz. Education. 2011;13:45-66.

18. Akbari B. Validity of motivation questionnaire of Heremence on students of high school in Guilan Providence. Knowledge Res Educ Sci. 2007;16:7396.

19. Weinberger S, Weeks W. The evolution of new business in health care. J Health Care Finance. 2004;31(2):53-61. PMID: 15839529

20. Zali M, Madhooshi M, Kerdnaeig A. Assess students' entrepreneurial characteristics. J Human Teach. 2007;81:81-113. 
21. Karimi A, Johari H. Identifying the Barriers to Entrepreneurship Development as Perceived by Graduate Students (Case: Faculties of Agriculture in Tehran Province). J Entrepreneursh Dev. 2013;6(4):151-67. DOI: 10.22059/jed.2013.50805

22. Azizi M, Zare H, Almasi H. The Correlation of Critical Thinking Disposition and Achievement Goal Orientation with Student Entrepreneurial Characteristics. Iranian J Med Educ. 2016;24(16):200-9.

23. Jahani S, Khoshknab F. Perceived entrepreneurial motivators by Iranian nurse entrepreneurs: a qualitative study. Q J Nurs Manage. 2014;3(3):68-77.

24. Philips F, Garman A. Barriers to entrepreneurship in health care organization. J Health Hum Serv Adm. 2006;28(4):472-84.

25. Soundy A, Muhamed A, Stubbs B, Probst M, Vancampfort D. The benefits of walking for individuals with schizophrenia spectrum disorders: A systematic review. Int $\mathbf{J}$ Ther Rehabil. 2014;21(9):410-20. DOI: 10.12968/ijtr.2014.21.9.410

26. Mohamadi E, Banaderakhshan H, Borhani F, Hoseinabadi Farahani M, Pour Hossiongholi M, Naderiravesh N. Relationship between Nursing Students' Achievement Motivation and Self-efficacy of Clinical Performance. Iran Univ Med Sci. 2014;27(90-91):33-43.

27. Sarmady M, Mahbooby T, Abdullah Zadeh H. Examine the relationship between self-esteem and achievement motivation and cognitive variables beliefs entrepreneurs in the provinces of East and West PNU students. J Psychol Univ Tabriz. 2009;4(14):1-27.

28. Dariyan M, Salehi M, khosravi Z. A comparative study on the motivation of progress in entrepreneurs or non-entrepreneurs in the industries of Tehran. $\mathrm{J}$ Entrepreneursh Dev. 2010;2(6):57-72. 